\title{
Dynamical Lamb Effect in a Tunable Superconducting Qubit-Cavity System
}

\author{
D. S. Shapiro ${ }^{1,2}$, A. A. Zhukov ${ }^{1,3}$, W. V. Pogosov ${ }^{1,4}$, Yu. E. Lozovik ${ }^{1,5,6}$ \\ ${ }^{1}$ Center for Fundamental and Applied Research, N. L. Dukhov \\ All-Russia Research Institute of Automatics, 127055 Moscow, Russia \\ ${ }^{2}$ V. A. Kotel'nikov Institute of Radio Engineering and Electronics, \\ Russian Academy of Sciences, 125009 Moscow, Russia \\ ${ }^{3}$ National Research Nuclear University (MEPhI), 115409 Moscow, Russia \\ ${ }^{4}$ Institute for Theoretical and Applied Electrodynamics, \\ Russian Academy of Sciences, 125412 Moscow, Russia \\ ${ }^{5}$ Institute of Spectroscopy, Russian Academy of Sciences, 142190 Moscow region, Troitsk, Russia and \\ ${ }^{6}$ Moscow Institute of Electronics and Mathematics, HSE, 101000 Moscow, Russia
}

\begin{abstract}
A natural atom placed into a cavity with time-dependent parameters can be parametrically excited due to the interaction with the quantized photon mode. One of the channels of such a process is the dynamical Lamb effect, induced by a nonadiabatic modulation of atomic level Lamb shift. However, in experiments with natural atoms it is quite difficult to isolate this effect from other mechanisms of atom excitation. We point out that a transmission line cavity coupled with a superconducting qubit (artificial macroscopic atom) provides a unique platform for the observation of the dynamical Lamb effect. A key idea is to exploit a dynamically tunable qubit-resonator coupling, which was implemented quite recently. By varying nonadiabatically the coupling, it is possible to parametrically excite a qubit through a nonadiabatic modulation of the Lamb shift, even if the cavity was initially empty. A dynamics of such a coupled system is studied within the Rabi model with time-dependent coupling constant and beyond the rotating wave approximation. An efficient method to increase the effect through the periodic and nonadiabatic switching of a qubit-resonator coupling energy is proposed.
\end{abstract}

PACS numbers: 42.50.Ct, 42.50.Dv, 85.25.Am

\section{INTRODUCTION}

Superconducting circuits with Josephson junctions can be used for quantum computing as qubits [1 6]. These systems are macroscopically large, but they demonstrate quantum behaviour, which allows them to be treated as artificial atoms. Superconducting qubits can be integrated with microwave waveguides, while the photon field in these waveguides is quantized. Such systems give rise to the effects of Rabi oscillations [7-10], quantum feedback [11, GHz photons emission [12, while many-qubit systems form subwavelength quantum metamaterials [13 16]. A well known Rabi model [17] is applicable for the description of qubit-photon quantum system [1].

Moreover, superconducting circuits integrated with microwave resonators provide a unique platform for an observation of cavity quantum electrodynamical (QED) nonstationary phenomena which can hardly be studied in more traditional experiments. This happens because (i) resonator frequencies in superconducting systems are nearly six orders of magnitude lower than frequencies in optical systems, (ii) superconducting qubit-cavity systems demonstrate high and fast tunability of their main parameters.

One of the examples of such nonstationary QED phenomena is the dynamical Casimir effect, predicted long time ago [18. According to the initial idea, an 'empty' space between two mirrors can emit photons due to vacuum fluctuations, provided these mirrors are rapidly moving with respect to each other. In order to observe this effect, one has to move mirrors with the speed approaching the speed of light, which seems to be unrealistic for a direct experimental realization. For this reason, there were several other proposals how the dynamical Casimir effect can be observed, see, e.g., Refs. [19 21]. In all these schemes, it was suggested that an effective boundary condition at 'mirrors' could be varied nonadiabatically, instead of moving massive 'mirrors' themselves. It is remarkable that it 
was a superconducting system, which led to the first observation of the dynamical Casimir effect [22, 23]. This was achieved by using an additional SQUID at the end of the waveguide in order to tune an effective boundary condition by varying in time the magnetic flux through the SQUID.

A presence of an additional atom in a cavity with time-dependent parameters leads to other nonstationary QED phenomena, since an atom and a photon field can interact with each other 24 28. For instance, an atom can be parametrically excited, even if the cavity was initially empty. It was shown in Ref. 27] that there are several channels for such a process. In the case of a nonadiabatical modulation of cavity parameters, there are two channels of such a process. The most obvious mechanism is due to the absorbtion of Casimir photons. Another channel is due to the nonadiabatic modulation of atomic Lamb shift: virtual states of atom-photon coupled system are transformed into real states. This phenomenon can be called a dynamical Lamb effect. Note that the existence of a static Lamb shift for superconducting qubit energy levels was verified experimentally in Ref. [29. In contrast to natural atoms, the effect can be significantly enhanced, since a strong coupling regime between the artificial macroscopic atom and the resonator is achievable.

In practice, it is quite difficult to isolate the mechanism of atom excitation due to the dynamical Lamb effect from another channel due to the the absorbtion of Casimir photons. In order to overcome this difficulty, it was suggested in Ref. 27] that a single natural atom can be passed through the resonator consisting of two cylindrical cameras of different diameters which are characterized by two unequal Lamb shifts. When leaving one camera and entering another one, the dynamical Lamb effect can occur, so that an atom can be parametrically excited. A specific feature of this scheme is that, in this case, no Casimir photon appears, so that the dynamical Lamb effect can be, in principle, isolated. Unfortunately, a direct experimental implementation of this idea is rather difficult.

The main goal of this paper is to suggest a superconducting qubit-resonator system as a suitable platform for the observation of the dynamical Lamb effect instead of a natural atom-cavity system. An astonishing property of superconducting qubits is a high tunability of their parameters in situ during an experiment [30, 31. For instance, one can modulate qubit excitation energy to manipulate qubits by using this degree of freedom [32. Moreover, due to a recent technological progress, it becomes realistic to vary dynamically not only the resonator frequency, but also a coupling between the artificial atom (transmon qubit) and photon field with the frequency of a resonator or even faster. This quantity already can be tuned in sufficiently wide ranges by an auxiliary SQUID, see, e.g., Ref. [33] and references therein. Using this additional degree of freedom, one can mimic a single atom passing from one camera to another one by a single switching of a qubit between the two resonators. Namely, this scheme can be mapped on a superconducting qubit at rest integrated with the resonator A, while at certain moment it is switched nonadiabatically to the initially empty resonator B. It is expected that this switching can be accompanied by the parametric excitation of a qubit. In this scheme, a channel of a qubit excitation through the absorbtion of real Casimir photons is suppressed, because in the weak coupling regime the perturbation of the cavity eigenmodes is negligible. Thus, instead of relying on a physical motion of an atom, one can simply tune a magnetic flux through a special auxiliary SQUID.

Actually, a high tunability of superconducting circuit systems allows even for a significant simplification of this mapping. Instead of utilizing two resonators, one can use a single one. Indeed, one can consider a qubit initially uncoupled from the resonator, while at certain moment this coupling is nonadiabatically switched on leading again to a possibility of a qubit excitation and photon generation. Furthermore, a superconducting qubit-resonator system can be made in a strong coupling regime which must increase dramatically the probability of qubit excitation due to the dynamical Lamb effect. Note that an important condition to observe the dynamical Lamb effect is also that all switchings have to be performed nonadiabatically. This implies that the typical switching time to be smaller than the resonator frequency. Such a regime becomes possible due to the very recent progress [33.

In order to describe theoretically the dynamical Lamb effect in the system with the tunable qubit-photon coupling, we use the Rabi model. To treat the dynamical Lamb effect, we have to go beyond the rotating wave approximation 34 (RWA) in order to take into account counter-rotating processes. These are the photon creation with the simultaneous qubit excitation and the opposite process. Such processes in superconducting qubit-resonator systems are not at all 
illusive, since they show up in experiments with strong-coupled systems, see, e.g., Ref. [35. We take into account counter-rotating wave terms by constructing a perturbation theory around the stationary RWA solution. In addition, we solve a time-dependent Schrödinger equation numerically. We find that our simple analytical formula for the qubit excitation probability gives excellent results in the case, when the interaction constant is switched nonadiabatically only once.

Our treatment does not only yield the description of the system's dynamics but also allows us to suggest a special trick which can be used to enhance a dynamical Lamb effect. Namely, we show that the qubit excitation probability can be dramatically increased provided that the coupling constant is switched on and off periodically with the period twice larger than the resonator frequency (each switching is nonadiabatical). Previously, similar periodic drivings have been proposed to enhance other cavity QED effects in a context of superconducting circuits, such as the dynamical Casimir effect [22, 23] or Lamb shift [36]. For such a periodic driving, our analytical perturbative result yields qualitatively correct description of the system's dynamics. We also examine the statistics of photon states, generated due to the coupling constant dynamics. We show that a significant squeezing of these states can be achieved. This result can be of practical importance.

We wish to stress that the main focus of this paper is not at all to develop a new method for superconducting qubit manipulation, but to suggest a scheme for the experimental realization of the dynamical Lamb effect. Note also that from the viewpoint of a quantum computing, the dynamical Lamb effect can be either positive or parasitic depending on a particular situation. For instance, it can lead to the undesirable qubit excitation. Therefore, the understanding and control of the dynamical Lamb effect is of importance both from the viewpoint of a fundamental physics and possible applications.

This paper is organized as follows. In Section II, we present our idea and outline a theoretical model used. In Section III, we find qubit excitation probability for different regimes of qubit-resonator coupling dynamics. In Section IV, we address correlation functions for both the photon field and qubit degrees of freedom upon coupling energy dynamics. We conclude in Section V.

\section{MODEL}

There are different types of superconducting qubits. All of them represent a superconducting ring containing several Josephson junctions, but they differ from each other by ratios of their charging energies and Josephson energies. In order to study experimentally the dynamical Lamb effect, we need a qubit which allows for the dynamical tunability between its own degrees of freedom and photon field with the frequency of a resonator or even faster. Actually, these are charge qubits (transmons) and flux qubits that seems to be more suitable for such a task 33.

The cavity is a coplanar waveguide where the photon mode frequency $\omega$ is large compared to the characteristic energy of interaction between the qubit and photon degrees of freedom (weak coupling). This limit supposes that the resonator is operated in the single mode regime. In practice, in order to enhance the dynamical Lamb effect, a strong coupling regime might be more appropriate. However, it is much more difficult to address this regime within the analytical treatment. We, therefore, focus on a weak coupling regime and show that even in this case a significant effect is possible.

The inductive coupling between a qubit and resonator photons is achieved by an auxiliary SQUID operating at the same GHz frequencies [33]. This opportunity is of a crucial importance for an observation of the dynamical Lamb effect. The coupling energy can be changed either by a single switching or via a periodic modulation. The latter approach will be shown to be much more efficient.

The qubit-resonator system can be described in terms of the Rabi model [17, 34, which is widely used in quantum optics. The total Hamiltonian of this model takes into account photons at the single mode $\omega$ and the qubit with the 
bare excitation frequency $\epsilon$ with the coupling $V$ between them

$$
H=\omega a^{\dagger} a+\frac{1}{2} \epsilon\left(1+\sigma_{3}\right)+V
$$

where $a^{\dagger}$ and $a$ are secondary quantized boson operators of the photon field, while Pauli matrices $\sigma_{3}=2 \sigma_{+} \sigma_{-}-1$, $\sigma_{+}, \sigma_{-}$act in the space of qubit states. The operator $V$ reads as

$$
V=g\left(a+a^{\dagger}\right)\left(\sigma_{-}+\sigma_{+}\right)
$$

where $\left(a+a^{\dagger}\right)$ and $\sigma_{-}+\sigma_{+}$, up to numerical factors, are nothing but the electric field and dipole moment operators, respectively, while $g \ll \omega$ is a coupling constant.Under this assumption, a perturbation of the cavity eigenmodes is negligible, so that a channel of qubit excitation via the absorbtion of real Casimir photons is not relevant.

A decoherence $\kappa$ and relaxation rate $\Gamma$ are of importance for real qubits, available in modern experiments. Typically, these two quantities turn out to be larger or of the same order as $g$. However, nowadays there is a steady and rather fast progress in decreasing $\kappa, \Gamma$, so we will concentrate on the limit $g \gg \kappa, \Gamma$.

In the case of a stationary system, a perturbation theory in operator $V$ can be developed rather easily. It was shown in Ref. 27] that $V$ produces contributions to the eigenenergies, which, in leading order, are independent on a photon level number but depend only on atomic level number. These contributions are interpreted as a Lamb shift, since these are virtual photons which are responsible for the effect. The approach of Ref. [27] is justified in the off-resonant regime $|\Delta| \gg g$, where $\Delta=\epsilon-\omega$ is a detuning frequency. It predicts the following shift for the atomic ground state energy:

$$
\epsilon_{L}=-\frac{g^{2}}{\omega+\epsilon} .
$$

Actually, there is another approach to the problem, which has some advantages. Namely, the operator $V$ can be split into the sum of two terms,

$$
V=V_{1}+V_{2}
$$

where

$$
V_{1}=g\left(a \sigma_{+}+a^{\dagger} \sigma_{-}\right)
$$

is the rotating wave contribution, conserving the excitation number, and

$$
V_{2}=g\left(a^{\dagger} \sigma_{+}+a \sigma_{-}\right)
$$

is the counter-rotating term, which breaks this symmetry, but conserves a parity.

We will focus on the situation, when the qubit-cavity system is near the resonance, $\epsilon \simeq \omega$, since in this case the dynamical Lamb effect is strongest. This resonant regime can be achieved by tuning qubit frequency $\epsilon$ in situ via an external magnetic flux, threading the qubit loop. Near the resonance and if $g$ is also independent on time, the fast oscillating term $V_{2}$ is usually omitted, while the resulting Hamiltonian is known to be exactly integrable. This well known solution is outlined in Appendix A. We have to take $V_{2}$ into account since it is precisely this operator, which is responsible for the stationary Lamb shift and, hence, for the dynamical Lamb effect as well. In the stationary case, $V_{2}$ can be treated as a perturbation. This approach is also utilized in Appendix A for the evaluation of the stationary Lamb shift in a resonant regime. Moreover, it will be demonstrated that a similar splitting of the Hamiltonian gives excellent results in certain nonstationary situations as well. 


\section{DYNAMICAL LAMB EFFECT}

\section{A. Qualitative picture}

This section deals with the main subject of our paper. Namely, we consider a coupled qubit-resonator system, when the coupling energy $g$ is time dependent. We assume that in the initial moment, the qubit and the resonator were not coupled, so that the initial state of the whole system was $|0 \downarrow\rangle$. When the coupling is switched on, higher-order photon states start to play their role, as well as a qubit excited state. In the case of a full resonance, this process can be illustrated by a scheme, presented in Fig. 1. Apart from the bare ground state $|0 \downarrow\rangle$, there are two sets of doubly-degenerate excited bare states. These states feel each other through the operator $V$, which is a sum of two contributions. The first one, $V_{1}$, conserves the excitation number, i.e., a total number of photons plus a qubit excitation number ( 0 or 1$)$. The second one, $V_{2}$, changes the excitation number by 2 by adding/removing one photon and simultaneously switching qubit to the excited/ground state.

Thus, we see that the bare ground state can be perturbed by $V_{2}$ only, which leads to the occupation of the $|1 \uparrow\rangle$ state with nonzero probability. In its turn, this latter state is connected by $V_{2}$ only with $|0 \downarrow\rangle$ and by $V_{1}$ only with $|2 \downarrow\rangle$. By continuing this process, we see that (i) only the states with even excitation number are populated, (ii) the states with the same excitation number are linked only via $V_{1}$, (iii) the states with the excitation numbers differing by 2 are linked by $V_{2}$ only.

Let us assume that tunable coupling $g$ is not oscillating too fast. In this case, $V_{2}$ must be responsible to fast oscillations, while $V_{1}$ corresponds to slow oscillations with frequencies essentially controlled by the Rabi frequency. Therefore, we may expect that $V_{2}$ can be treated perturbatively, although both $V_{1}$ and $V_{2}$ are formally proportional to the same parameter $g$ (a small parameter responsible for this feature in a resonant case is $g / \omega$ ). This implies that the qubit excitation probability is going to experience oscillations with a frequency of the order of the Rabi frequency. Such oscillations are induced by processes between the states of the same excitation number. By solving time-dependent Schrödinger equation numerically, we will also show that this qualitative picture is indeed correct. It is also of importance to note that the photon-qubit dynamical coupling results in the occupation of all photon states, i.e., with both odd and even photon numbers. This feature, which can be probed in experiments, is in contrast with the dynamical Casimir effect, which leads to the occupation of the states with only even number of photons [27].

Notice that slow Rabi-like oscillations show up even in the first order of the perturbation theory around the RWA solution. In contrast, when applying a perturbation theory around bare states, all terms of an infinite expansion in $V$ must be taken into account in order to recover such oscillations. This is one of the advantages of the theoretical approach utilized in the present paper.

\section{B. Time-dependent perturbation theory}

As a zero-order approximation, we take the stationary RWA Hamiltonian, in which the coupling constant is equal to the time average value $\bar{g}=\langle g(t)\rangle_{t}$ (averaging is performed after the coupling is switched on) and remaining nonstationary terms are treated as a perturbation. This perturbation contains counter rotating wave operator $V_{2}$ and a rotating wave contribution, which is due to deviations of $g$ from its time averaged value. Namely, the full Hamiltonian is represented as

$$
H(t)=H_{R W A}+V(t),
$$

where

$$
\begin{array}{r}
H_{R W A}=\omega a^{\dagger} a+\frac{1}{2} \epsilon\left(1+\sigma_{3}\right)+\bar{g}\left(a \sigma_{+}+a^{\dagger} \sigma_{-}\right), \\
V(t)=g(t)\left(a^{\dagger} \sigma_{+}+a \sigma_{-}\right)+(g(t)-\bar{g})\left(a \sigma_{+}+a^{\dagger} \sigma_{-}\right) .
\end{array}
$$




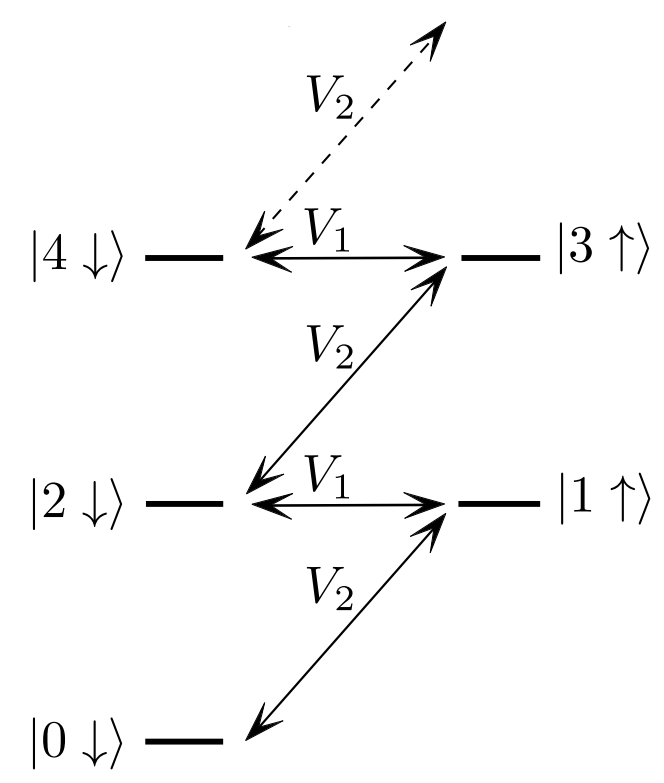

FIG. 1: A scheme illustrating an internal structure of the full wave function projected into states of the qubit-waveguide system without coupling (see in the text).

Let us now consider the evolution of the wave function $\psi(t=0)=|0 \downarrow\rangle$ assuming that a coupling is switched on at $t=0$. Then, at the moment $t>0$, the wave function is given by the standard expression

$$
\psi(t)=e^{-i H_{R W A} t} \mathcal{T} \exp \left(-i \int_{0}^{t} e^{i H_{R W A} \tau} V(\tau) e^{-i H_{R W A} \tau} d \tau\right) \psi(0),
$$

where $\mathcal{T}$ stands for the time ordering operation. In our calculations we take into account only first order terms in $\mathcal{T}$-exponent series expansion in Eq. (9). This approximation is further justified by a direct comparison with the result of the solution of the time-dependent Schrödinger equation.

\section{Driving by a single switching of a coupling constant}

We now study the evolution of the wave function in a full resonance regime, $\epsilon=\omega$, after the sudden switch of the coupling $g$, which then stays constant in time. Thus, we have $g(t)=g \theta(t)$ and $\bar{g}=g$, so that the second contribution in operator $V(t)$, as given by Eq. (8), vanishes. Using Eq. (9), we obtain

$$
\psi(t)=|0 \downarrow\rangle-\frac{g}{2 \omega}\left(e^{-2 i \omega t} \cos \sqrt{2} g t-1\right)|1 \uparrow\rangle-i \frac{g}{2 \omega} e^{-2 i \omega t} \sin \sqrt{2} g t|2 \downarrow\rangle .
$$

In this approximation, the qubit excitation probability as a result of the instantaneous switching, is given by

$$
w_{\uparrow}(t)=\frac{g^{2}}{8 \omega^{2}}(3+\cos 2 \sqrt{2} g t-4 \cos 2 \omega t \cos \sqrt{2} g t) .
$$

This probability corresponds to the dynamical Lamb effect and includes both slow and fast oscillations. In the superconducting systems with weak coupling between photon field and qubit, the probability is as small as $g^{2} / \omega^{2}$. Note that the amplitude of this quantity is proportional to the square of the change of the static Lamb shift, in accordance with Ref. 27.

The number of generated photons as a function of time $n_{p h}(t)=\left\langle\psi(t)\left|a^{\dagger} a\right| \psi(t)\right\rangle$ can be also readily extracted from Eq. (10). We would like to stress that these photons appear as a result of the dynamical Lamb effect. Our results for $w_{\uparrow}(t)$ and $n_{p h}(t)$ at $\omega / g=20$ are plotted in Fig. 2 for $0<t<1.2 T_{R}$, where the Rabi period is defined as $T_{R}=\pi / g$. 
In order to justify these results, we also solved Schrödinger equation numerically in the weak coupling regime. The basis was truncated by typically 10 photon states. An accuracy was verified by increasing the number of such states and comparison with results for a smaller basis. The results of such numerical computations show excellent agreement with our perturbative results for both $w_{\uparrow}(t)$ and $n_{p h}(t)$. The relative discrepancy is essentially within few percent. Such an excellent agreement justifies our qualitative understanding of the system dynamics, discussed above.
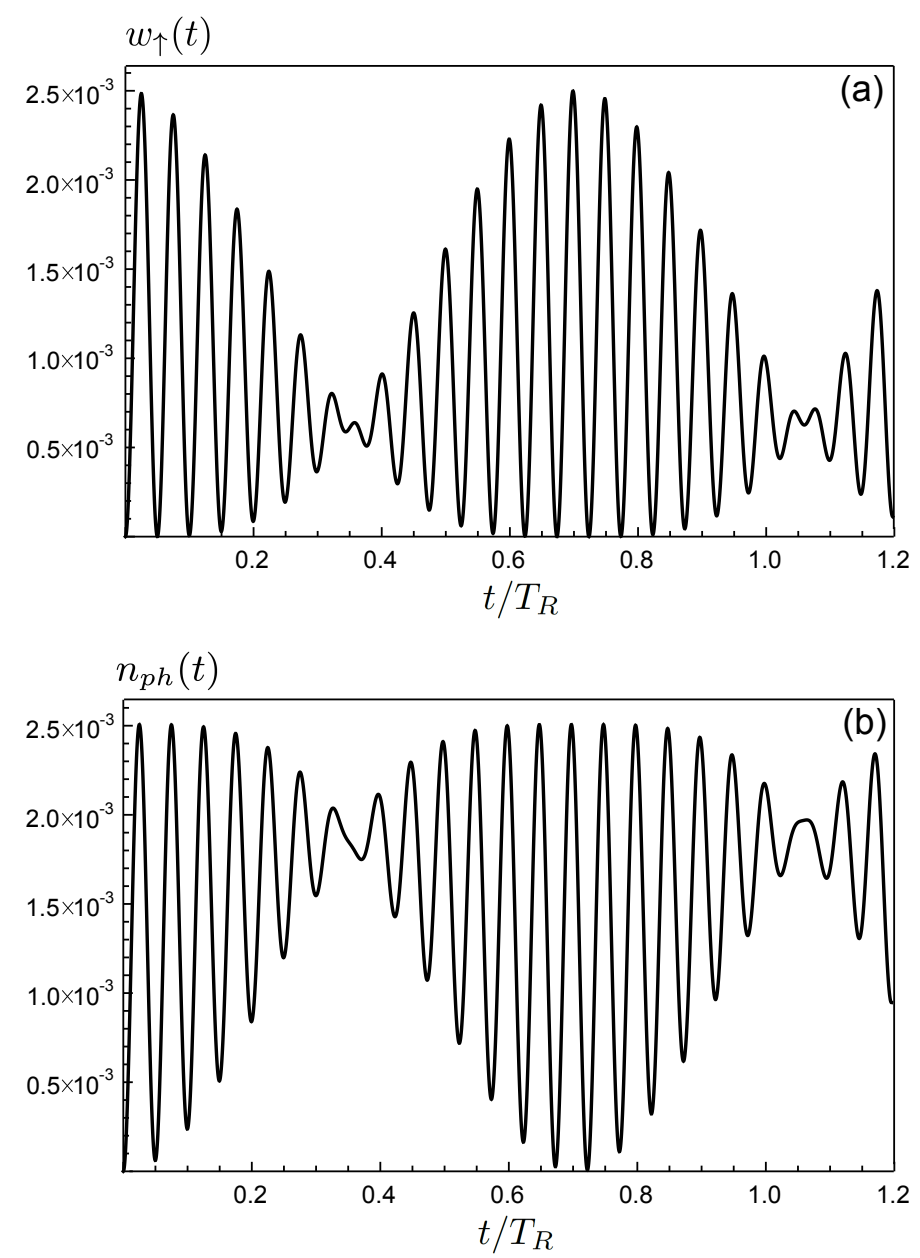

FIG. 2: The qubit excitation probability $w_{\uparrow}(t)$ (a) and the photon number $n_{p h}(t)$ (b) as functions of time after a single switching of the qubit-waveguide coupling constant at $\omega / g=20$.

\section{Parametric driving}

Single switching of the qubit-resonator coupling results in the low probability for the qubit excitation. In order to enhance it, one can use more sophisticated modulations of $g$ as a function of time. We, however, should keep in mind that the dynamical Lamb effect can be isolated only in the case, when this coupling changes nonadiabatically, so that the only one way is to switch it off and on instantaneously.

The excitation probability can be dramatically increased at the regime of a parametric resonance, when the driving is induced by high frequency periodic switching of the qubit-resonator coupling. In particular, we suggest applying $2 \omega$-periodical switching on and off, namely, $g(t)=g \theta(\cos 2 \omega t)$. Hence, we take $\bar{g}=g / 2$ in the Hamiltonian splitting, as given by Eq. (8). In order to evaluate qubit excitation probability, we again limit ourselves to the first order approximation in Eq. (9). 
We again assume that $\omega \gg g$. Therefore, fast oscillating terms of the form $e^{i \omega t} g(t)$ can be replaced by their average values $\left\langle e^{i \omega t} g(t)\right\rangle_{t}$, when performing integration in Eq. 9 , while corrections to this approximation are negligible as $g / \omega$. Under this replacement, we find

$$
\psi(t) \simeq|0 \downarrow\rangle+\frac{2}{\pi} e^{-2 i \omega t}\left(\left(1-\cos \frac{g t}{\sqrt{2}}\right)|2 \downarrow\rangle-i \sin \frac{g t}{\sqrt{2}}|1 \uparrow\rangle\right) .
$$

The corresponding excitation probability is

$$
w_{\uparrow}(t) \simeq \frac{2}{\pi^{2}}(1-\cos \sqrt{2} g t) .
$$

This result describes an approximate Rabi-like oscillatory behavior of the qubit excitation probability via the dynamical Lamb effect in the regime of a parametric resonance. Note that frequences of oscillations of qubit excitation probability and mean photon number differ by a factor of 2, according to Eq. 12. Regardless of the relation between amplitude of the switching $g$ and $\omega$, the maximum probability reaches the finite value $4 / \pi^{2}$ on a characteristic time scale $\sim 1 / g$, which depends on the amplitude of the inverse coupling strength only. In contrast to the probability in the regime of a single switching (11), the time dependence of $w_{\uparrow}(t)$ in parametric resonance regime 13 does not contain high-frequency oscillations at $\omega$. Moreover, the maximum probability is not small anymore. This last feature is of great importance for the possibility of an experimental investigation of the dynamical Lamb effect.

We also wish to stress that the precise value of the maximum excitation probability, as given by Eq. (11), is rather relative. Namely, if we take into account the next term of the perturbative expansion in Eq. (9), the prefactor $2 / \pi^{2}$ is going to change, staying nevertheless independent on $g$. In addition, an admixture of Rabi-like harmonic with higher frequency will appear, which corresponds to oscillations between the two states with the number of excitations equal to 4 . Nevetheless, Eq. 13 yields correct qualitative description for $w_{\uparrow}(t)$, as further evidenced by a comparison with fully numerical results. A lack of a quantitative agreement is due to the fact that our picture based on the separation of fast and slow degrees of freedom is not so accurate anymore because of a high-frequency modulation of $g$.

Next, we solve the time dependent Schrödinger equation numerically. We indeed see Rabi-like oscillations for both the qubit excitation probability and the mean photon number, as shown by Fig. 3. Frequencies of these oscillations are correctly described by Eq. 13 . The estimated period of $w_{\uparrow}(t)$ oscillations corresponds to the time scale $\sqrt{2 \pi} / g$ appearing in the RWA Hamiltonian in the two-excitation solution. We also found that maximum $w_{\uparrow}(t)$ and photon number $n_{p h}(t)$ are only slightly sensitive to the amplitude of the coupling strength $g$ in the weak interaction regime, which is again in a qualitative agreement with the simple analytical treatment. At the same time, the value of maximum $w_{\uparrow}(t)$, as predicted by Eq. [13), is nearly twice underestimated compared to the exact (numerical) solution. Thus, both $w_{\uparrow}(t)$ and $n_{p h}(t)$ demonstrate a universal behavior. Namely, by rescaling the time, they can be cast in a universal form within a few percent accuracy. This feature, which is predicted qualitatively by Eq. (13), is in a full agreement with the results of our numerical computations.

Recently, it was suggested theoretically that the Lamb shift of a qubit can be greatly enhanced via periodical driving of a qubit by the external classical electromagnetic field [36]. This idea has some similarities with our idea of parametric pumping. However, in contrast to Ref. [36], we do not need an external classical field which directly affects a qubit and modifies its Lamb shift. Instead we modulate only the qubit-resonator coupling, while qubit is excited and photons appear as a result of the interaction between a qubit and resonator.

Nevertheless, both ideas indicate that counter-rotating processes in a weak coupling regime can be significantly enhanced by using various types of a periodic driving. The same strategy was used to increase a dynamical Casimir effect which finally has led to its experimental observation [22, 23]. Thus, periodic driving, in general, can be exploited to enhance various nonstationary cavity QED effects. 

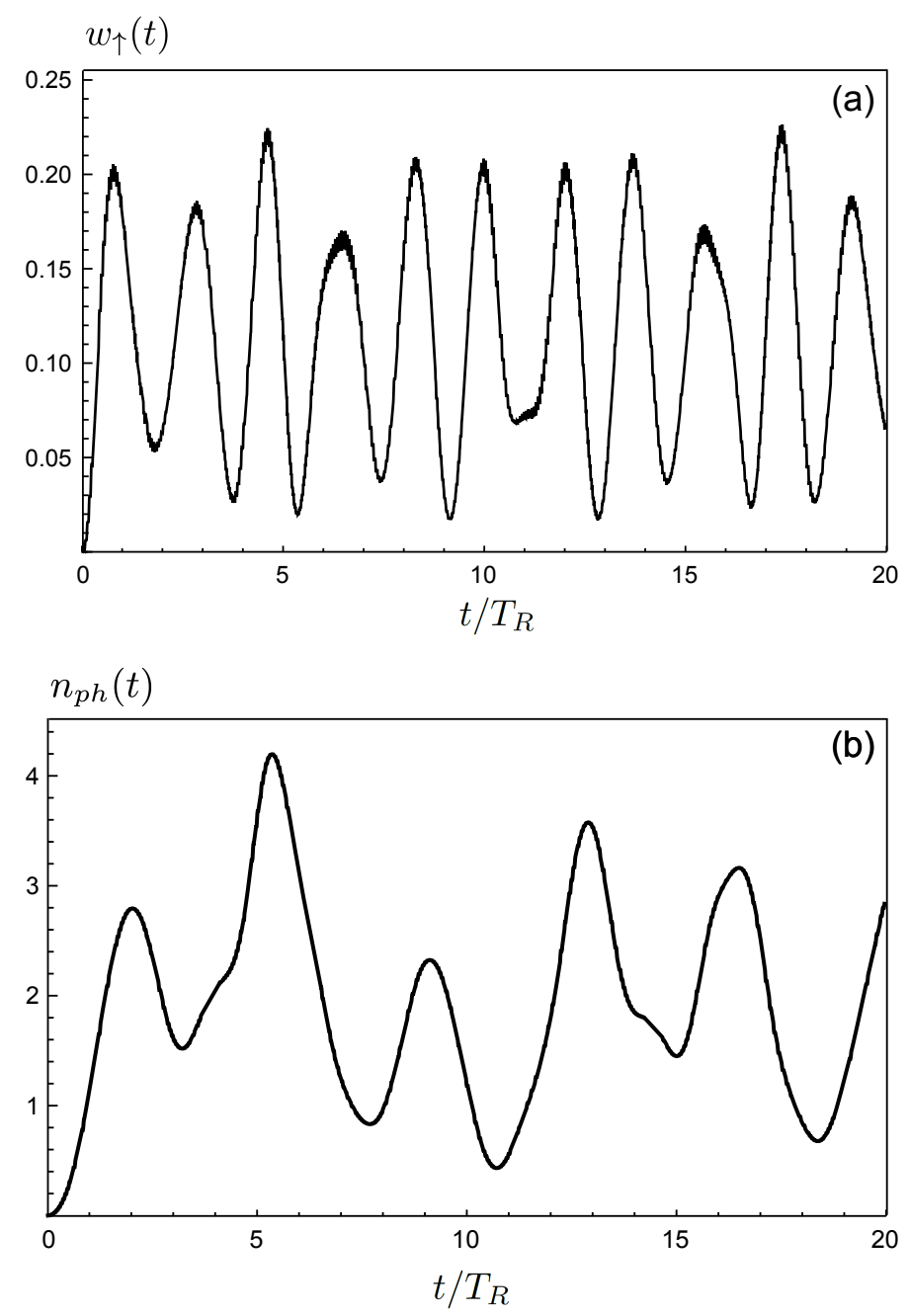

FIG. 3: The qubit excitation probability $w_{\uparrow}(t)(\mathrm{a})$ and the photon number $n_{p h}(t)$ (b) as functions of time after the beginning of parametric driving at $\omega / g=20$.

\section{CORRELATION FUNCTIONS}

In addition to the qubit degrees of freedom, our system has also photon ones. They can be characterized by various correlation functions. For instance, one can use squeezing parameters defined in a standard way as $\Delta p \equiv$ $\frac{1}{2} \sqrt{\left\langle\left(a-a^{\dagger}\right)^{2}\right\rangle-\left\langle a-a^{\dagger}\right\rangle^{2}}$ and $\Delta x \equiv \frac{1}{2} \sqrt{\left\langle\left(a+a^{\dagger}\right)^{2}\right\rangle-\left\langle a+a^{\dagger}\right\rangle^{2}}$. For the stationary system, these two parameters can be evaluated approximately using a perturbation theory around RWA solution (see Appendix A). The wave function $\psi_{0}^{(1)}$ yields the following squeezing parameters (in the first order by $g$ )

$$
\Delta p=\Delta x=\frac{1}{2}\left(1-\frac{2 g \alpha_{2}^{(-)} \beta_{2}^{(-)}}{\epsilon_{2, \psi}}\right) .
$$

It is easy to see that $\Delta p, \Delta x>1 / 2$, since $\alpha_{2}^{(-)} \beta_{2}^{(-)}<0$, which implies that this state is not squeezed.

Let us now consider squeezing in the case of a parametric modulation of the qubit-resonator coupling constant. An accuracy of the analytical solution in the first order of perturbation theory is not sufficient for the quantitative analysis. We therefore restrict ourselves to the numerical solution of the time dependent Schrödinger equation taking into account nearly 30 photon states. Numerical solution shows the realization of the squeezed state under parametric driving of a particular form $g(t)=g \theta(\cos (2 \omega t))$. We observe non-periodic oscillations of the squeezing parameter $\Delta x$. 


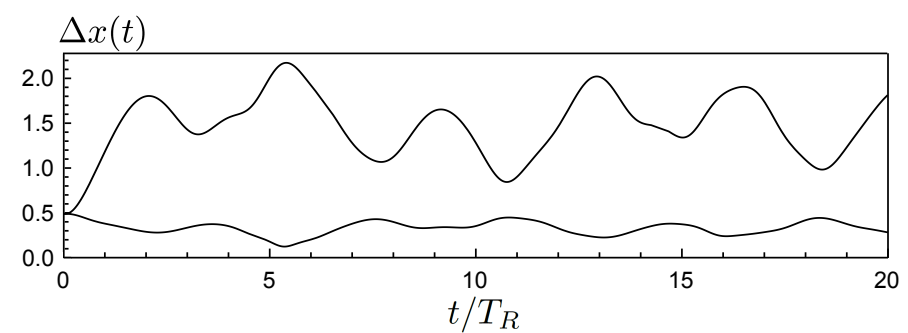

FIG. 4: Numerical result for the squeezing parameter $\Delta x(t)$ at $\omega / g=20$ within 20 Rabi periods after the beginning of the parametric driving. Only lower and upper envelope curves are shown, while $\Delta x(t)$ experiences fast oscillations between them.

Figure 4 shows the lower and upper envelope curves for the evolution of $\Delta x$ within first 20 Rabi periods after the switching on the driving $g(t)$ for $\omega / g=20$. These envelope curves experience slow oscillations, while fast oscillations of $\Delta x$ occur between them. The fast oscillations are not shown in Fig. 4.

The results again reveal the universality of the envelope curves oscillations, regardless of the ratio $\omega / g$. The minimum value of $\Delta x$ at particular moments of time, according to Fig. 4 , is $\approx 1 / 4$, which indicates a sufficiently strong squeezing of a generated photon state. By comparison Fig. 4 and Fig. 3, we see that oscillations of $\Delta x$ are correlated with oscillations of $n_{p h}(t)$.
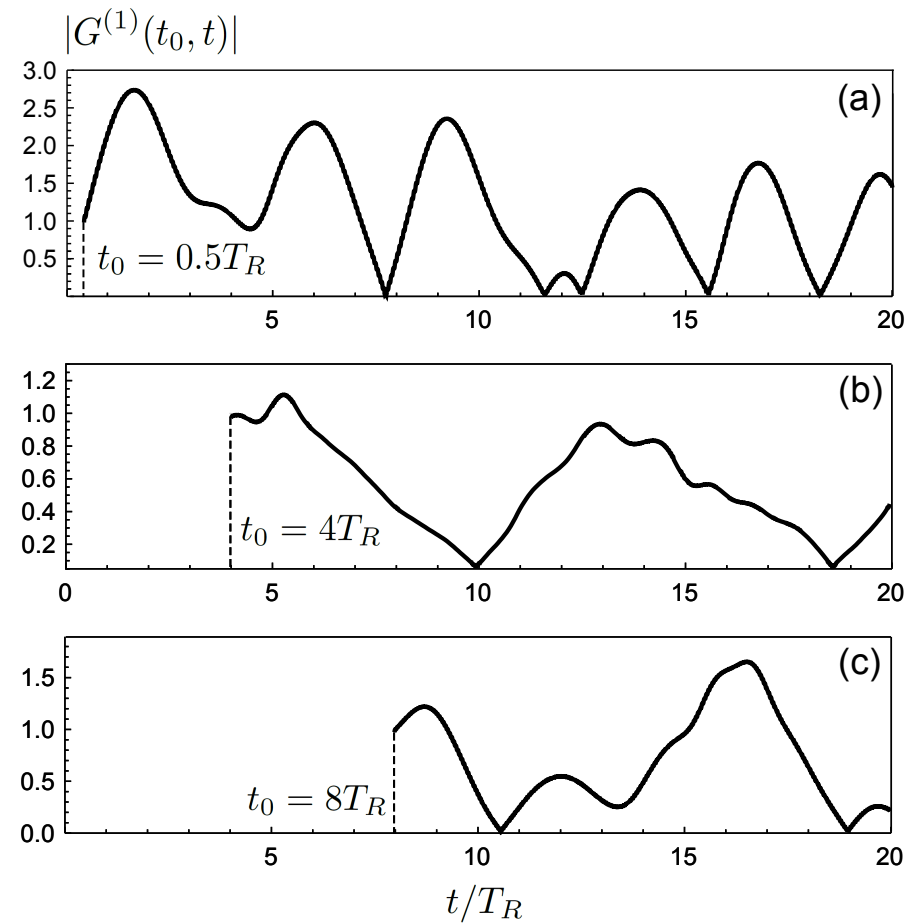

FIG. 5: Numerical result for photon correlation functions $G^{(1)}\left(t_{0}, t\right)$ at $t_{0}=0.5 T_{R}$ (a), $4 T_{R}$ (b), $8 T_{R}$ (c) at $\omega / g=20$ within 20 Rabi periods after the starting of the parametric driving.

The first-order and second-order correlation functions can be expressed through the field operators in a Heisenberg picture as

$$
\begin{gathered}
G^{(1)}\left(t_{0}, t\right)=\frac{\left\langle a^{\dagger}\left(t_{0}\right) a(t)\right\rangle}{\left\langle a^{\dagger}\left(t_{0}\right) a\left(t_{0}\right)\right\rangle}, \\
G^{(2)}\left(t_{0}, t\right)=\frac{\left\langle a^{\dagger}\left(t_{0}\right) a\left(t_{0}\right) a^{\dagger}(t) a(t)\right\rangle}{\left\langle a^{\dagger}\left(t_{0}\right) a\left(t_{0}\right) a^{\dagger}\left(t_{0}\right) a\left(t_{0}\right)\right\rangle} .
\end{gathered}
$$

These two quantities are calculated using a numerical solution of the time-dependent Schrödinger equation. Typical 

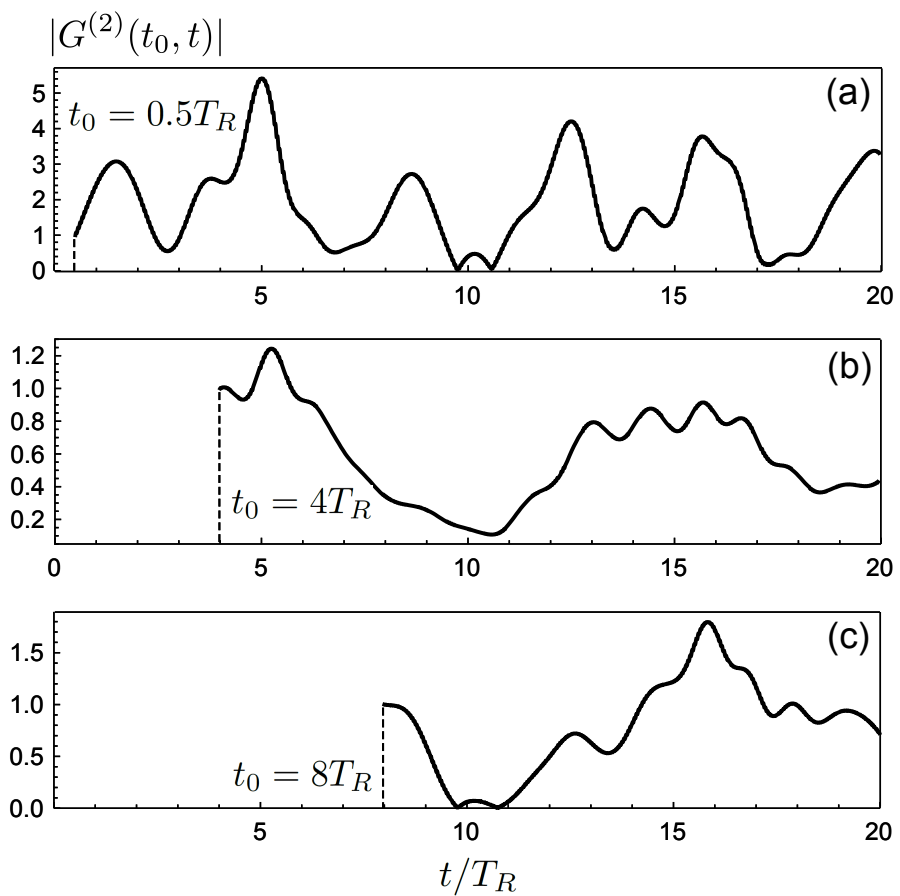

FIG. 6: Numerical result for photon correlation functions $G^{(2)}\left(t_{0}, t\right)$ at $t_{0}=0.5 T_{R}$ (a), $4 T_{R}$ (b), and $8 T_{R}$ (c) at $\omega / g=20$ within 20 Rabi periods after the starting of the parametric driving.

results are presented in Figs. 5 and 6. Both correlation functions demonstrate non-periodic oscillations with slow decay as functions of $t-t_{0}$, where $t_{0}$ corresponds to the starting point and the correlation is probed at $t>t_{0}$. By comparing these two figures with Fig 3 , it is easy to see that the correlation functions at small $t_{0}$ generally follow the field revivals as a function of $t$, while oscillations are not so pronounced at larger $t_{0}$. The existence of slow oscillations is clearly linked to the interplay between the qubit and photon field, which is accompanied by the qubit excitation due to the dynamical Lamb effect.

We also introduce first-order and second-order correlation functions for qubit degrees of freedom (qubit excitation number) as

$$
\begin{gathered}
G_{q}^{(1)}\left(t_{0}, t\right)=\frac{\left\langle\sigma_{+}\left(t_{0}\right) \sigma_{-}(t)\right\rangle}{\left\langle\sigma_{+}\left(t_{0}\right) \sigma_{-}\left(t_{0}\right)\right\rangle}, \\
G_{q}^{(2)}\left(t_{0}, t\right)=\frac{\left\langle\sigma_{+}\left(t_{0}\right) \sigma_{-}\left(t_{0}\right) \sigma_{+}(t) \sigma_{-}(t)\right\rangle}{\left\langle\sigma_{+}\left(t_{0}\right) \sigma_{-}\left(t_{0}\right) \sigma_{+}\left(t_{0}\right) \sigma_{-}\left(t_{0}\right)\right\rangle},
\end{gathered}
$$

which are again calculated numerically. The results are shown in Figs. 7 and 8 (for the same set of parameters as for Figs. 5 and 6). The behavior of these correlation functions generally resembles the behavior of similar quantities for the photon field. The dynamics of $G_{q}^{(1)}$ and $G_{q}^{(2)}$ follows the dynamics of $w_{\uparrow}(t)$.

However, the amplitude of oscillations of $G_{q}^{(2)}$ is rather small, while this quantity does not experience a decay as a function of $t-t_{0}$. This is related to the two-level character of qubit energy spectrum which has to be contrasted with the unbounded photon spectrum. Moreover, for the considered range of parameters, photons occupy several levels, while qubit mostly stays in its ground state. This latter feature leads to only small oscillations of $G_{q}^{(2)}$. Nevertheless, slow oscillations of correlation functions for the photon field, which can be probed in experiments, are definitely related to slow oscillations of correlation functions for qubit degrees of freedom, as well as to the qubit excitation probability.

Thus, slow Rabi-like oscillations of various characteristics of a photon field can be used in experiments as a signature of the dynamical Lamb effect in the systems with tunable photon-qubit coupling. Indeed, if the resonator was initially empty and qubit was uncoupled from it, no such oscillations can exist, of course. If a coupling energy is modulated, qubit can be excited leading to photon creation via counter-rotating processes, which can be seen in experiments. In 

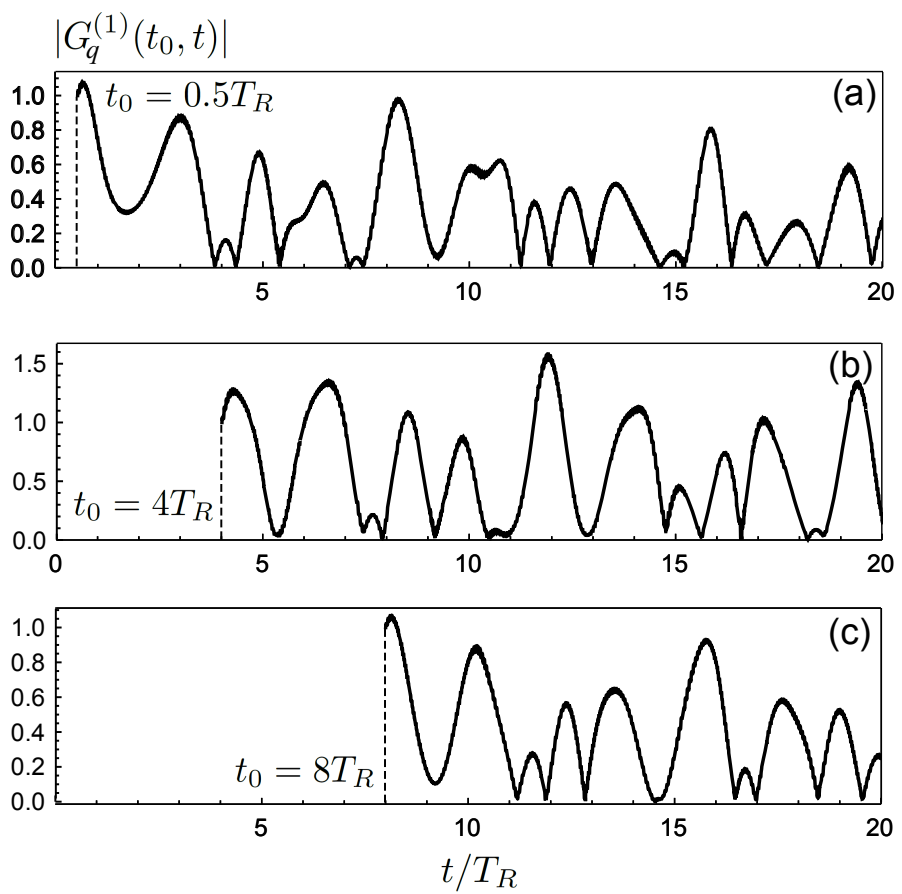

FIG. 7: Numerical result for qubit correlation functions $G_{q}^{(1)}\left(t_{0}, t\right)$ at $t_{0}=0.5 T_{R}(\mathrm{a}), 4 T_{R}(\mathrm{~b}), 8 T_{R}(\mathrm{c})$ at $\omega / g=20$ within 20 Rabi periods after the starting of the parametric driving.

contrast to the dynamical Casimir effect, photons occupy not only even states.

\section{CONCLUSIONS}

We suggested that a coupled superconducting qubit-resonator system can be used for the observation of the dynamical Lamb effect. This effect was initially introduced for the natural atom in a varying cavity [27. It leads to the probability for the atom to be excited solely due to the nonadiabatic modulation of atomic levels Lamb shift. Although this effect was initially predicted for natural atoms, it is quite problematic to observe it in experiments with such atoms, since this effect must be carefully separated from other mechanisms of atom excitation. To this end, it was proposed in Ref. 27] to pass a single atom through the resonator consisting of two cameras with different diameters. When passing the cameras, an atom can be excited and a photon can be generated. We suggest a nontrivial link between this scheme and a system with a single superconducting qubit (macroscopic artificial atom) at rest integrated with the resonator in such a way that the coupling can be tuned dynamically. By switching on this coupling nonadiabatically, one can excite a qubit and generate a photon, even if the resonator was initially unoccupied by photons. Such a dynamical coupling between the qubit (transmon) and the resonator is already achievable by utilizing an auxiliary SQUID 33. Thus, we suggest that this scheme can be used for the experimental realization of the dynamical Lamb effect.

We presented a theoretical model for the description of the system dynamics upon a modulation of the coupling energy and for the predictions of a method to increase the effect. Our treatment is based on the dynamical Rabi model in which we take into account counter-rotating terms, since they are responsible for the dynamical Lamb effect. The dynamical problem was solved both analytically using a perturbation theory around the RWA stationary solution and numerically by integrating time dependent Schrödinger equation.

We found that a single switching of the coupling constant results only in a low probability for the qubit to be excited when considering weak coupling regime, as well as a full resonance between the qubit and the cavity single 

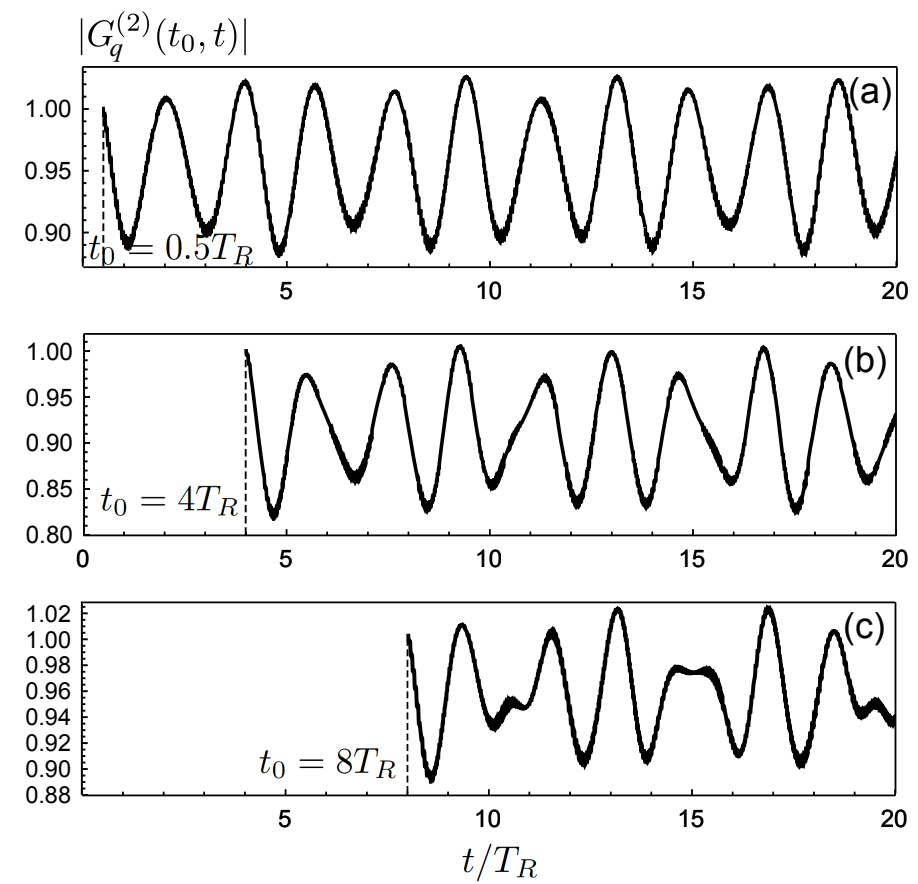

FIG. 8: Numerical result for qubit correlation functions $G_{q}^{(2)}\left(t_{0}, t\right)$ at $t_{0}=0.5 T_{R}(\mathrm{a}), 4 T_{R}(\mathrm{~b})$, and $8 T_{R}(\mathrm{c})$ at $\omega / g=20$ within 20 Rabi periods after the starting of the parametric driving.

mode. After the switching on, this probability experiences slow Rabi-like oscillations. We then suggested a much more efficient method to enhance the effect which is a periodic nonadiabatical switching of the coupling energy. We also studied a statistics of photon states generated upon the coupling constant modulation. We found that these states can experience a strong squeezing.

\section{Acknowledgments}

The authors acknowledge useful comments by A. V. Ustinov, A. L. Rakhmanov, V. P. Yakovlev, and L. V. Bork. This work was supported by RFBR (project no. 15-02-02128). W. V. P. acknowledges the support from the Russian Science Support Foundation. Yu. E. L. is supported by Program of Basic Research of HSE.

\section{Appendix A: Perturbation theory around RWA solution}

The stationary Rabi Hamiltonian can be split as

$$
H=H_{R W A}+V_{2} .
$$

The first part $H_{R W A}$ is the Hamiltonian in the rotating wave approximation,

$$
H_{R W A}=\omega a^{\dagger} a+\frac{1}{2} \epsilon\left(1+\sigma_{3}\right)+V_{1},
$$

which is known to be exactly integrable.

The set of the "dressed" eigenstates and corresponding eigenenergies of $H_{R W A}$ is given by the following expressions

$$
\begin{array}{ll}
\psi_{n}^{(0)}=\alpha_{n}^{(-)}|n \downarrow\rangle+\beta_{n}^{(-)}|n-1 \uparrow\rangle, & \epsilon_{n, \psi}^{(0)} \\
\chi_{n}^{(0)}=\alpha_{n}^{(+)}|n \downarrow\rangle+\beta_{n}^{(+)}|n-1 \uparrow\rangle, & \epsilon_{n, \chi}^{(0)}
\end{array}
$$


where $|n \downarrow\rangle$ and $|n-1 \uparrow\rangle$ form a set of "bare" states of the decoupled qubit and photons. Coefficients in A3 read as $(n \geq 1)$ :

$$
\begin{aligned}
& \alpha_{n}^{( \pm)}=\frac{g \sqrt{n}}{\sqrt{\frac{\Delta^{2}}{2}+2 g^{2} n \pm \Delta \sqrt{\frac{\Delta^{2}}{4}+g^{2} n}}} \\
& \beta_{n}^{( \pm)}=\frac{\frac{\Delta}{2} \pm \sqrt{\frac{\Delta^{2}}{4}+g^{2} n}}{\sqrt{\frac{\Delta^{2}}{2}+2 g^{2} n-\Delta \sqrt{\frac{\Delta^{2}}{4}+g^{2} n}}} .
\end{aligned}
$$

A bare ground state $|0 \downarrow\rangle$ is not affected by $V_{1}$ and remains the same as at $g=0$. Thus, there is only one state corresponding to $n=0$, which is $\psi_{0}^{(0)}$ :

$$
\psi_{0}^{(0)}=|0 \downarrow\rangle .
$$

The eigenenergies of $H_{R W A}$ are

$$
\begin{array}{ll}
\epsilon_{n, \psi}=\omega n+\frac{\Delta}{2}-\sqrt{\frac{\Delta^{2}}{4}+g^{2} n}, \quad n \geq 0, \\
\epsilon_{n, \chi}=\omega n+\frac{\Delta}{2}+\sqrt{\frac{\Delta^{2}}{4}+g^{2} n}, \quad n \geq 1 .
\end{array}
$$

Using standard perturbation theory, we find that the wave functions within the first order contribution in $V_{2}$ read $(n \geq 1)$

$\psi_{n}^{(1)}=\psi_{n}^{(0)}+g\left(\frac{\alpha_{n-2}^{(-)} \beta_{n}^{(-)} \sqrt{n-1}}{\epsilon_{n, \psi}-\epsilon_{n-2, \psi}} \psi_{n-2}^{(0)}+\frac{\beta_{n+2}^{(-)} \alpha_{n}^{(-)} \sqrt{n+1}}{\epsilon_{n, \psi}-\epsilon_{n+2, \psi}} \psi_{n+2}^{(0)}+\frac{\alpha_{n-2}^{(+)} \beta_{n}^{(-)} \sqrt{n-1}}{\epsilon_{n, \psi}-\epsilon_{n-2, \chi}} \chi_{n-2}^{(0)}+\frac{\beta_{n+2}^{(+)} \alpha_{n}^{(-)} \sqrt{n+1}}{\epsilon_{n, \psi}-\epsilon_{n+2, \chi}} \chi_{n+2}^{(0)}\right)$.

$\chi_{n}^{(1)}=\chi_{n}^{(0)}+g\left(\frac{\alpha_{n-2}^{(+)} \beta_{n}^{(+)} \sqrt{n-1}}{\epsilon_{n, \chi}-\epsilon_{n-2, \chi}} \chi_{n-2}^{(0)}+\frac{\beta_{n+2}^{(+)} \alpha_{n}^{(+)} \sqrt{n+1}}{\epsilon_{n, \chi}-\epsilon_{n+2, \chi}} \chi_{n+2}^{(0)}+\frac{\alpha_{n-2}^{(-)} \beta_{n}^{(+)} \sqrt{n-1}}{\epsilon_{n, \chi}-\epsilon_{n-2, \psi}} \psi_{n-2}^{(0)}+\frac{\beta_{n+2}^{(-)} \alpha_{n}^{(+)} \sqrt{n+1}}{\epsilon_{n, \chi}-\epsilon_{n+2, \psi}} \psi_{n+2}^{(0)}\right)$.

For the modified ground state $(n=0)$, we obtain

$$
\psi_{0}^{(1)}=|0 \downarrow\rangle-\frac{g \beta_{2}^{(-)}}{\epsilon_{2, \psi}}\left(\alpha_{2}^{(-)}|2 \downarrow\rangle+\beta_{2}^{(-)}|1 \uparrow\rangle\right) .
$$

Using the above strategy, one can also find corrections in $V_{2}$ to the eigenstates of $H_{R W A}$. For instance, a first non-zero contribution to the ground state energy is determined by the second order correction and is given by the negative value

$$
\epsilon_{0, \psi}^{(2)}=-\frac{g^{2}\left|\beta_{2}^{(-)}\right|^{2}}{\epsilon_{2, \chi}}=\frac{-4 g^{2}\left(1-\Delta / \sqrt{\Delta^{2}+8 g^{2}}\right)}{3 \omega+\epsilon-\sqrt{\Delta^{2}+8 g^{2}}} .
$$

This is the Lamb shift value, where all the processes, generated by the rotating wave term $V_{1}$ in the Hamiltonian, are included in zero-order approximation. This scheme of the expansion with respect to the operator $V_{2}$ around $H_{R W A}$ regularizes the perturbation theory against $1 / \Delta$ divergencies. It is applicable in the opposite resonant case $|\Delta| \ll g$, where the perturbative result of Ref. [27] is not adequate due to divergent corrections $\sim g / \Delta$ to the wave functions and eigen energies. The divergencies originate from the degeneracy of the self-mode and qubit energy. 
It is of importance to note that Eq. (A9) does not match the off-resonant result $(3)$ that means that this $V_{2^{-}}$ expansion is most reliable in the resonant limit $\Delta \ll g$, despite that the detuning $\Delta$ is formally not restricted in this approach. In order to recover the off-resonant result (3) we have to consider contributions from higher orders of $V_{2}$.

[1] P. D. Nation, J. R. Johansson, M. P. Blencowe, and F. Nori, Rev. Mod. Phys. 84, 1 (2012).

[2] J. Q. You and F. Nori, Nature 474, 589 (2011).

[3] A. Blais, R.-S. Huang, A. Wallraff, S. M. Girvin, and R. J. Schoelkopf, Phys. Rev. A 69, 062320 (2004).

[4] T. P. Orlando, J. E. Mooji, L. Tian, C. H. van der Wal, L. Levitov, S. Lloyd, and J. J. Mazo, Science, 285, 1036 (1999).

[5] D. I. Schuster, A. A. Houck, J. A. Schreier, A. Wallraff, J. M. Gambetta, A. Blais, L. Frunzio, B. Johnson, M. H. Devoret, S. M. Girvin, and R. J. Schoelkopf, Nature 445, 515 (2007).

[6] E.O. Kiktenko, A.K. Fedorov, O.V. Man'ko, and V.I. Man'ko, Phys. Rev. A 91, 042312 (2015).

[7] R. Vijay, C. Macklin, D. H. Slichter, S. Weber, K. Murch, R. Naik, A. N. Korotkov, and I. Siddiqi, APS March Meeting (Boston MA, Feb. 27 - March 2, 2012), Bulletin of APS, 57, 1, (2012).

[8] J. M. Fink, R. Bianchetti, M. Baur, M. Goppl, L. Steffen, S. Filipp, P. J. Leek, A. Blais, and A. Wallraff, Phys. Rev. Lett. 103, 083601 (2009).

[9] P. Bertet, I. Chiorescu, G. Burkard, K. Semba, C. J. P. M. Harmans, D. P. Di Vincenzo, and J. E. Mooij, Phys. Rev. Lett. 95, 257002 (2005).

[10] A. Wallraff, D. I. Schuster, A. Blais, L. Frunzio, J. Majer, M. H. Devoret, S. M. Girvin, and R. J. Schoelkopf, Phys. Rev. Lett. 95, 060501 (2005).

[11] C. Sayrin, I. Dotsenko, X. Zhou, B. Peaudecerf, T. Rybarczyk, S. Gleyzes, P. Rouchon, M. Mirrahimi, H. Amini, M. Brune, J.-M. Raimond, and S. Haroche, Nature 477, 73 (2011).

[12] G. Romero, J. J. Garcia-Ripoll, and E. Solano, Phys. Rev. Lett. 102, 173602 (2009).

[13] A. L. Rakhmanov, A. M. Zagoskin, S. Savelev, and F. Nori, Phys. Rev. B 77, 144507 (2008).

[14] O. Astafiev, A. M. Zagoskin, A. A. Abdumalikov Jr., Yu. A. Pashkin, T. Yamamoto, K. Inomata, Y. Nakamura, and J. S. Tsai, Science 327, 840 (2010).

[15] P. Macha, G. Oelsner, J.-M. Reiner, M. Marthaler, S. André, G. Schön, U. Hübner, H.-G. Meyer, E. Ilichev, and A. V. Ustinov, Nat. Commun. 5, 5146 (2014).

[16] D. S. Shapiro, P. Macha, A. N. Rubtsov, and A. V. Ustinov, Photonics 2, 449 (2015).

[17] I. I. Rabi, Phys. Rev. 49, 324 (1936); 51, 652 (1937).

[18] G. T. Moore, J. Math. Phys. 11, 2679 (1970).

[19] E. Yablonovich, Phys. Rev. Lett. 62, 1742 (1989).

[20] Yu. E. Lozovik, V. G. Tsvetus, and E. A. Vinogradov, Phys. Scr. 52, 184 (1995).

[21] A. V. Dodonov, E. V. Dodonov, and V. V. Dodonov, Phys. Lett. A 317, 378 (2003).

[22] P. Lähteenmäki, G. S. Paraoanu, J. Hassel, and P. J. Hakonen, Proc. Natl. Acad. Sci. U.S.A. 110, 4234 (2013).

[23] C. M. Wilson, G. Johansson, A. Pourkabirian, J. R. Johansson, T. Duty, F. Nori, and P. Delsing, Nature 479, 376 (2011).

[24] D.J. Heinzen and M.S. Feld, Phys. Rev. Lett. 59, 2623 (1987).

[25] A. A. Belov, Yu. E. Lozovik, and V. L. Pokrovski, Sov. Phys. JETP 96, 552 (1989).

[26] A. M. Fedotov, N. B. Narozhny, and Yu. E. Lozovik, Phys. Lett. A 274, 213 (2000).

[27] N. B. Narozhny, A. M. Fedotov, and Yu. E. Lozovik, Phys. Rev. A 64, 053807 (2001).

[28] H. Walther, B. T. H. Varcoe, B. G. Englert, and T. Becker, Rep. Prog. Phys. 69, 1325 (2006).

[29] A. Fragner, M. Goppl, J. M. Fink, M. Baur, R. Bianchetti, P. J. Leek, A. Blais, and A. Wallraff, Science, 322, 1357 (2008).

[30] M. H. Devoret, S. Girvin, and R. J. Schoelkopf, Ann. Phys. (Leipzig) 16, 767 (2007).

[31] R. J. Schoelkopf and S. Girvin, Nature 451, 664 (2008).

[32] J. D. Strand, M. Ware, F. Beaudoin, T. A. Ohki, B. R. Johnson, A. Blais, and B. L. T. Plourde, Phys. Rev. B 87, 220505(R) (2013).

[33] Yu Chen, C. Neill, P. Roushan, N. Leung, M. Fang, R. Barends, J. Kelly, B. Campbell, Z. Chen, B. Chiaro, A. Dunsworth, E. Jeffrey, A. Megrant, J. Y. Mutus, P. J. J. OMalley, C. M. Quintana, D. Sank, A. Vainsencher, J. Wenner, T. C. White, M. R. Geller, A. N. Cleland, and J. M. Martinis, Phys. Rev. Lett. 113, 220502 (2014). 
[34] E. T. Jaynes and F. W. Cummings, Proc. IEEE 51, 89 (1963).

[35] T. Niemczyk, F. Deppe, H. Huebl, E. P. Menzel, F. Hocke, M. J. Schwarz, J. J. Garcia-Ripoll, D. Zueco, T. Hummer, E. Solano, A. Marx, and R. Gross, Nat. Phys. 6, 772 (2010).

[36] V. Gramich, S. Gasparinetti, P. Solinas, and J. Ankerhold, Phys. Rev. Lett. 113, 027001 (2014); V. Gramich, P. Solinas, M. Möttönen, J. P. Pekola, and J. Ankerhold, Phys. Rev. A 84, 052103 (2011). 